PROCEEDINGS OF THE

AMERICAN MATHEMATICAL SOCIETY

Volume 134, Number 9, September 2006, Pages 2639-2648

S 0002-9939(06)08287-6

Article electronically published on March 22, 2006

\title{
A LANDING THEOREM FOR PERIODIC RAYS OF EXPONENTIAL MAPS
}

\author{
LASSE REMPE
}

(Communicated by Juha M. Heinonen)

\begin{abstract}
For the family of exponential maps $z \mapsto \exp (z)+\kappa$, we show the following analog of a theorem of Douady and Hubbard concerning polynomials. Suppose that $g$ is a periodic dynamic ray of an exponential map with nonescaping singular value. Then $g$ lands at a repelling or parabolic periodic point. We also show that there are periodic dynamic rays landing at all periodic points of such an exponential map, with the exception of at most one periodic orbit.
\end{abstract}

\section{INTRODUCTION}

In polynomial dynamics, dynamic rays, which foliate the basin of the superattracting fixed point $\infty$, play an important role. By a result of Douady and Hubbard, every periodic dynamic ray of a polynomial with connected Julia set lands at a repelling or parabolic periodic point [M1, Theorem 18.10]. The combinatorial information given by this fact was a key element of their initial study of the Mandelbrot set [DH], and has since proved to be one of the most fundamental tools in the field.

In the case of exponential maps $E_{\kappa}(z)=\exp (z)+\kappa$, the point $\infty$ is no longer an attracting fixed point but rather an essential singularity, and the set of escaping points is no longer open. However, it has long been known [DGH] that there exist curves of escaping points in the dynamical plane which can be seen as an analog of dynamic rays (see Section 2). The combinatorics of such rays landing together has also been used to great advantage in this family (see, e.g., [S2, RS1, RS2]).

Therefore it is a natural and important question whether periodic dynamic rays of exponential maps land in general; compare [S1, Section VI.6]. In this article, we give a positive answer to this question.

Theorem 1. Let $\kappa \in \mathbb{C}$ such that the singular value $\kappa$ of $E_{\kappa}$ does not escape to infinity. Then every periodic dynamic ray of $E_{\kappa}$ lands at a repelling or parabolic periodic point.

Remark. For maps with escaping singular value, there is an obvious exception if the singular value lies on a periodic dynamic ray (and this is the only exception). Since these parameters were already treated in SZ2, we have formulated the theorem for nonescaping parameters to simplify its statement.

Received by the editors July 30, 2003 and, in revised form, March 30, 2005.

2000 Mathematics Subject Classification. Primary 37F10; Secondary 30D05.

(C)2006 American Mathematical Society

Reverts to public domain 28 years from publication 
The analog of Theorem 1 in the polynomial case is proved by a hyperbolic contraction argument. In the exponential family, this proof can be modified to work in the special case where the ray considered does not intersect the postsingular set [SZ2, R1]. This condition is satisfied for all periodic dynamic rays in the important cases of attracting, parabolic and postsingularly finite parameters; see [SZ2]. For other conditions under which the landing of periodic rays can be proved by an argument in the dynamical plane, compare R3, R5.

On the other hand, there is a generic subset of the bifurcation locus consisting of maps whose postsingular set is the entire plane [R1, Theorem 5.1.6]. In this case, it is impossible to prove by hyperbolic contraction that a given periodic ray lands, unless one can a priori ensure that the ray does not accumulate on the singular value $\kappa$. However, the accumulation behavior of dynamic rays can be very complicated in general, e.g., for a large set of parameters there exist rays whose accumulation sets are indecomposable continua [R4]. It even seems difficult to show dynamically, without any structural assumptions, that the accumulation set of a periodic ray cannot be the entire plane.

Our proof of Theorem 1 manages to circumvent these problems by working in the parameter plane, using the " $\lambda$-lemma" from the theory of holomorphic motions MSS and a theorem of Schleicher concerning the landing of periodic parameter rays S1. It is somewhat surprising that the landing of dynamic rays is proved using landing properties of parameter rays. Unfortunately, this method will not carry over to higher-dimensional parameter spaces of entire transcendental functions.

We also prove a partial converse to Theorem 11. In the case of a polynomial with connected Julia set, it is known that every repelling or parabolic periodic point is the landing point of a dynamic ray [M1, Theorem 18.11]. The analog of this theorem has previously been shown for attracting, parabolic, escaping and postsingularly finite exponential maps [SZ2]. (In the escaping case, there will again be exceptions when the singular value lies on a periodic ray.)

Theorem 2. Let $\kappa \in \mathbb{C}$ such that the singular value $\kappa$ of $E_{\kappa}$ does not escape. Then, with the exception of at most one periodic orbit, every periodic point of $E_{\kappa}$ is the landing point of a periodic dynamic ray.

An irrationally indifferent periodic point can never be the landing point of a periodic dynamic ray by the Snail Lemma [M1, Lemma 16.2]. Thus our theorem implies that, in the case where $\kappa$ is a Siegel or Cremer parameter, all repelling periodic points are landing points. We believe that, as for polynomials, nonrepelling orbits are the only exceptions which can actually occur in Theorem 2, This conjecture can be phrased as a question about parameter space (see Section 5) and would imply that every indifferent parameter $\kappa$ is the landing point of a parameter ray.

Organization of the article. Naturally, we will rely on a fair number of results now established in the theory of exponential maps. We have attempted to collect these in Sections 2 and 4 , so that the proofs of Theorems 1 and 2 will only rely on the propositions stated there.

Notation and preliminaries. Throughout this article, $\mathbb{C}$ will denote the complex plane, and all closures will be taken in $\mathbb{C}$ (rather than on the Riemann sphere). The set of escaping points of an exponential map $E_{\kappa}$ is denoted by

$$
I\left(E_{\kappa}\right):=\left\{z \in \mathbb{C}:\left|E_{\kappa}^{n}(z)\right| \rightarrow \infty\right\} .
$$


By $[\mathrm{EL}$, an exponential map has at most one periodic orbit which is not repelling. Therefore, we call a parameter $\kappa$ attracting, resp. parabolic, if $E_{\kappa}$ has an attracting, resp. parabolic (i.e., rationally indifferent), periodic orbit. We also fix the function $F:[0, \infty) \rightarrow[0, \infty), F(t)=\exp (t)-1$ as a model for exponential growth.

The completion of a proof is marked by the symbol $\boldsymbol{\square}$. Results which are cited without proof are concluded by $\square$.

\section{DyNAMIC AND PARAMETER RAYS OF EXPONENTIAL MAPS}

2.1. Dynamic rays. We call a sequence $\underline{s}=s_{1} s_{2} s_{3} \ldots$ of integers an external address. We say that a point $z \in \mathbb{C}$ has external address $\underline{s}$ if

$$
\operatorname{Im}\left(E_{\kappa}^{n-1}(z)\right) \in\left(\left(2 s_{n}-1\right) \pi,\left(2 s_{n}+1\right) \pi\right)
$$

for every $n \geq 1$. The shift map on external addresses is denoted by $\sigma$; i.e. $\sigma\left(s_{1} s_{2} s_{3} \ldots\right)=s_{2} s_{3} s_{4} \ldots$. For simplicity, we will restrict to bounded external addresses; i.e. those for which the sequence $\left(s_{k}\right)$ of entries is bounded. For the results in the general case, see [SZ1] or [R2].

Proposition 1 ([SZ1]). Let $\kappa \in \mathbb{C}$, and let $\underline{s}$ be a bounded external address. If $T>0$ is large enough, then there exists a unique curve $g:[T, \infty) \rightarrow I\left(E_{\kappa}\right)$ such that

(a) $g(t)$ has external address $\underline{s}$ for all $t \geq T$, and

(b) $\operatorname{Re}\left(E_{\kappa}^{n}(g(t))\right)=F^{n}(t)+o(1)$ as $n$ or $t$ tend to $\infty$.

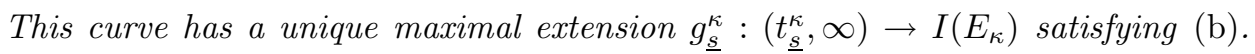
This extension is injective and will be called the dynamic ray of $E_{\kappa}$ at address $\underline{s}$.

The family of dynamic rays has the following additional properties:

- $E_{\kappa}\left(g_{\underline{s}}^{\kappa}\right) \subset g_{\sigma(\underline{s})}^{\kappa}$ for all $\underline{s}$;

- for every $\underline{s}, t_{\underline{s}}^{\kappa}$ depends upper semicontinuously on $\kappa$;

- for every $t>0, g_{\underline{s}}^{\kappa}(t)$ depends analytically on $\kappa$ (where defined);

- $t_{\underline{s}}^{\kappa} \neq 0$ if and only if there exists $n \geq 1$ with $\kappa \in g_{\sigma^{n}(\underline{s})}^{\kappa}$ (in which case we call $g_{\underline{s}}^{\kappa}$ broken).

Remark. In the case of unbounded addresses, the number 0 in the last item is replaced by a number $t_{\underline{s}} \geq 0$ which depends only on $\underline{s}$.

As usual, we say that a dynamic ray $g_{\underline{s}}^{\kappa}$ lands if $t_{\underline{s}}^{\kappa}=0$ and $\lim _{t \rightarrow 0} g_{\underline{s}}^{\kappa}(t)$ exists. In our proofs of Theorems 1 and 2 , we will use the fact, mentioned in the Introduction, that these theorems are known to hold for attracting and parabolic parameters.

Proposition 2 ([SZ2, Theorem 3.2 and Theorem 5.4]). Let $\kappa$ be an attracting or parabolic parameter. Then every periodic dynamic ray of $E_{\kappa}$ lands at a repelling or parabolic periodic point. Conversely, every repelling or parabolic periodic point of $E_{\kappa}$ is the landing point of a periodic dynamic ray.

Remark. In fact, for these parameters even more is true: every dynamic ray lands, and every point in the Julia set is either on a ray or the landing point of a ray BDD, R2]. 
Parameter rays. By [S1, Theorem II.8.1] (which was recently generalised to the case of unbounded addresses; see [F, FS, FRS]), the set

$$
G_{\underline{s}}:=\left\{\kappa \in \mathbb{C}: \kappa \in g_{\underline{s}}^{\kappa}\left(\left(t_{\underline{s}}^{\kappa}, \infty\right)\right)\right\}
$$

is itself a continuous (and differentiable) curve to $\infty$ for every bounded external address $\underline{s}$. This curve is called the parameter ray at address $\underline{s}$. We shall require the following result of Schleicher, stating that every periodic parameter ray lands. (The ray period of a parabolic parameter $\kappa$ is the period of the repelling petals of $E_{\kappa}$ at the parabolic orbit; in particular, it is a multiple of the period of this orbit.)

Proposition 3 ([S1, Theorem V.7.2]). Suppose that $\underline{s}$ is a periodic address of period $n$. Then $\overline{G_{\underline{s}}} \backslash G_{\underline{s}}$ consists of a single parabolic parameter of ray period $n$.

Conversely, for every parabolic parameter $\kappa$ of ray period $n$, there exists a periodic address of period $n$ such that $\kappa \in \overline{G_{\underline{s}}}$.

Schleicher's proof of this proposition consists of two steps. First it is shown that every parabolic parameter is the landing point of one or two parameter rays (whose addresses depend on the combinatorics of the parameter $\kappa$ ). This result is so far unpublished outside of S1. It is then shown (independently from the previous step) that, for every periodic address $\underline{s}$, there exists a parabolic parameter with the required combinatorics. A proof of this can be found in [RS1, Theorem 8.5].

Vertical order. Finally, let us note that the vertical order of dynamic rays coincides with the lexicographic order $<$ on external addresses. More precisely, let $\underline{s} \neq \underline{r}$ be bounded external addresses, and let $t_{0}>t_{\underline{s}}^{\kappa}$. If $R>0$ is large enough, then the set $\{z: \operatorname{Re} z>R\} \backslash g_{\underline{s}}^{\kappa}\left(\left[t_{0}, \infty\right)\right)$ has two unbounded components $U^{+}$and $U^{-}$, with unbounded positive, respectively negative, imaginary parts. The ray $g_{\underline{r}}$ tends to $\infty$ in $U^{-}$if and only if $\underline{r}<\underline{s}$. (This fact follows easily from the definition of dynamic rays; see [R1, Lemma 3.7.1] or [FS, Lemma 3.9] for details.)

This statement can be transferred to the parameter plane [FS, Proposition 3.10]. That is, if $\underline{s}^{1}$ and $\underline{s}^{2}$ are bounded external addresses, then $G_{\underline{s}^{1}}$ is below $G_{\underline{s}^{2}}$ in the above sense if and only if $\underline{s}^{1}<\underline{s}^{2}$.

\section{LANDING OF PERIODIC DYNAMIC RAYS}

The key idea in our proof of Theorem 1 is that dynamic rays move holomorphically with respect to the parameter (where defined). By the " $\lambda$-lemma" from MSS, the same is true for their closure. For completeness, we include the short proof of this fact (which is analogous to the argument for the general case in [MSS]).

Theorem 3. Let $\underline{s}$ be a bounded external address. Let $W$ be a connected component of the set

$$
\mathbb{C} \backslash \overline{\bigcup_{n \geq 1} G_{\sigma^{n}(\underline{s})}} .
$$

Suppose furthermore that for some $\kappa_{0} \in W, g_{\underline{s}}^{\kappa_{0}}$ lands. Then $g_{\underline{s}}^{\kappa}$ lands for every $\kappa \in W$, and the landing point depends holomorphically on $\kappa$.

Remark. The theorem (and its proof) remains true for arbitrary external addresses.

Proof. Let $z_{0}$ be the landing point of $g_{\underline{s}}^{\kappa_{0}}$. By definition of $W$, the ray $g_{\underline{s}}^{\kappa}$ is unbroken (i.e., $t_{\underline{s}}^{\kappa}=0$ ) for every $\kappa \in W$. We shall use the notation $h_{t}(\kappa):=g_{\underline{s}}^{\kappa}(t)$. The family $\left(h_{t}\right)_{t \in(0,1)}$ of holomorphic functions is normal by Montel's theorem, as it omits 


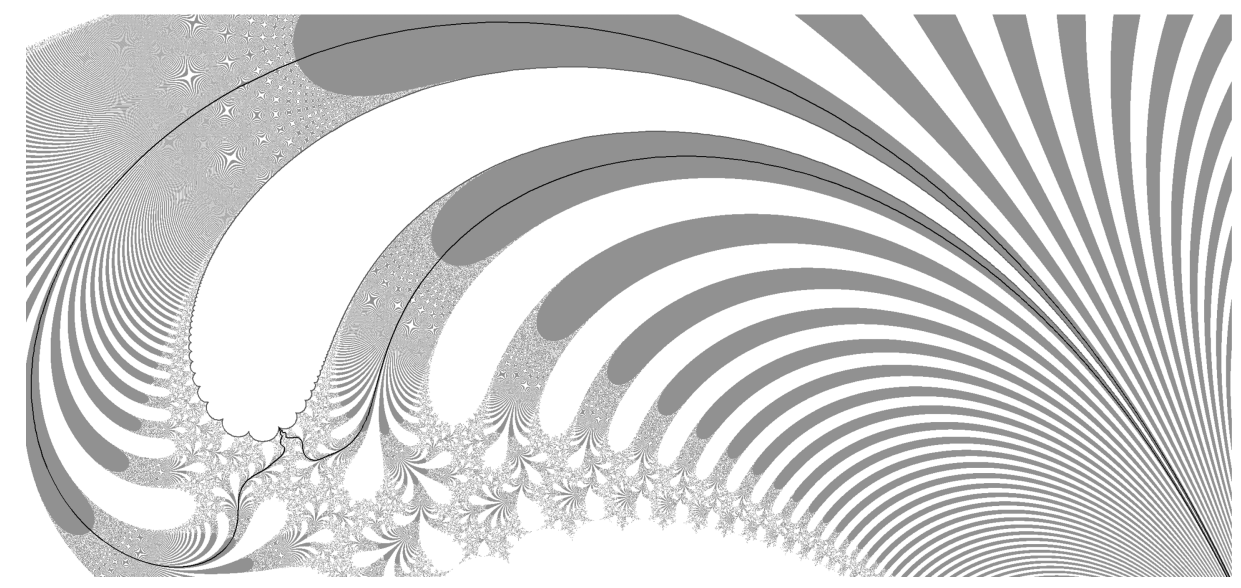

Figure 1. The wake of a period 5 hyperbolic component.

the holomorphic functions $\kappa \mapsto \kappa$ and $\kappa \mapsto h_{1}(\kappa)$. Let $h_{0}$ be a limit function of this family as $t \rightarrow 0$. Note that $h_{0}\left(\kappa_{0}\right)=z_{0}$ and thus, by Hurwitz's theorem, $h_{0}(\kappa) \neq h_{t}(\kappa)$ for all $\kappa \in W$ and $t>0$.

Now if $\widetilde{h_{0}}$ is some other limit function of $h_{t}$ as $t \rightarrow 0$, then $\widetilde{h_{0}}\left(\kappa_{0}\right)=z_{0}=h_{0}\left(\kappa_{0}\right)$. Since the $h_{t}$ omit the holomorphic function $h_{0}$, we can apply Hurwitz's theorem once more to see that $\widetilde{h_{0}}=h_{0}$. In other words, $h_{t} \rightarrow h_{0}$ as $t \rightarrow 0$. In particular, $g_{\underline{s}}^{\kappa}(t) \rightarrow h_{0}(\kappa)$ for every $\kappa \in W$.

Proof of Theorem 1. Let $\underline{s}$ be any periodic address of period $n$, and let $\kappa \in \mathbb{C}$ be a parameter with $\kappa \notin I\left(E_{\kappa}\right)$. Set

$$
K:=\bigcup_{1 \leq j \leq n} \overline{G_{\sigma^{j}(\underline{s})}} .
$$

By Propositions 3 and 2, we may suppose that $\kappa \notin K$.

Let $W$ be the connected component of $\mathbb{C} \backslash K$ containing $\kappa_{0}$. It is a standard fact that $W$ contains some attracting parameter $\kappa_{0}$. (This follows from Proposition 4 below, but can be proved in a much more elementary way: by [S2, Theorem 3.5], there is a hyperbolic component of parameter space (compare Section 4) tending to $\infty$ between any two parameter rays.) The ray $g_{\underline{s}}^{\kappa_{0}}$ lands by Proposition 2 , and by Theorem 3, the ray $g_{\underline{s}}^{\kappa}$ also lands. Its landing point is repelling or parabolic by the Snail Lemma [M1, Lemma 16.2].

\section{WAKES OF HYPERBOLIC COMPONENTS}

The article [RS2] gives a complete description of the combinatorial structure of wakes in exponential parameter space. Proposition 3 and Theorem 3 can be used to translate these results into statements about the regions in which certain periodic rays land together, which will be needed in the proof of Theorem 2 . These results, which are all analogs of well-established results for the Mandelbrot set (see, e.g., [M2]), will be given in this section. Their proofs necessarily involve citing several combinatorial results from [RS2].

A hyperbolic component $W$ is a maximal connected subset of parameter space in which all maps have an attracting periodic orbit, the period of which is necessarily 
constant on $W$. It is well known $[\mathrm{BR}]$ that there is a unique hyperbolic component of period one, which contains the left half-plane $\{\operatorname{Re} \kappa<-1\}$.

Proposition 4. Let $W$ be a hyperbolic component of period $n>1$. Then there exists a unique parabolic parameter $\kappa_{0} \in \partial W$ of ray period $n$ (called the root of $W$ ) which is the common landing point of two periodic parameter rays $G_{\underline{s}^{1}}$ and $G_{\underline{s}^{2}}$ of period $n$. These rays are called the characteristic rays of $W$, and they separate $W$ from the unique hyperbolic component of period one. The connected component of

$$
\mathbb{C} \backslash\left(G_{\underline{s}^{1}} \cup\left\{\kappa_{0}\right\} \cup G_{\underline{\underline{s}}^{2}}\right)
$$

which contains $W$ is called the wake of $W$ and denoted by $\mathcal{W}(W)$. Any two periodic parameter rays which land at a common point are the characteristic rays of some hyperbolic component.

Remark. The unique period one component does not have characteristic rays; by definition, its wake is all of $\mathbb{C}$.

For a proof of Proposition 4, compare Definition 8.6 and the preceding discussion in RS1. The wake of a hyperbolic component is important to us because configurations of periodic dynamic rays landing together are preserved throughout it. In order to make this precise, suppose that $z_{0}$ is a periodic point of period $n$ for an exponential map $E_{\kappa_{0}}$ and $U$ is an open neighborhood of $\kappa_{0}$ in parameter space. By an analytic extension of $z_{0}$ on $U$ we mean an analytic function $z: U \rightarrow \mathbb{C}$ with $z\left(\kappa_{0}\right)=z_{0}$ and $E_{\kappa}^{n}(z(\kappa))=z(\kappa)$ for all $\kappa \in U$. By the implicit function theorem and the identity principle, $z_{0}$ has at most one analytic extension on $U$ provided that $\left(E_{\kappa}^{n}\right)^{\prime}\left(z_{0}\right) \neq 1$. Furthermore, if $U$ is simply connected and contains no parabolics whose ray period divides $n$, then $z_{0}$ does have an analytic extension to $U$ by the monodromy theorem.

Proposition 5. Let $W$ be a hyperbolic component and $\kappa_{0} \in W$. Suppose that $E_{\kappa_{0}}$ has a periodic point $z_{0}$ which is the landing point of two different periodic dynamic rays $g_{s^{1}}^{\kappa_{0}}$ and $g_{s^{2}}^{\kappa_{0}}$. Then $z_{0}$ has an analytic extension $z$ to $\mathcal{W}(W)$, and for all $\kappa \in \mathcal{W}(W), g_{\underline{s}^{1}}^{\kappa}$ and $g_{\underline{s}^{2}}^{\kappa}$ land at $z(\kappa)$.

Proof. By [S2, Lemma 5.2], there exist two periodic addresses $\underline{r}^{-}<\underline{r}^{+}$such that

(a) $g_{\underline{r}^{-}}^{\kappa}$ and $g_{\underline{r}^{+}}^{\kappa}$ land at a common point belonging to the orbit of $z_{0}$, and

(b) for all $k \geq 0$ and each $j \in\{1,2\}, \sigma^{k}\left(\underline{s}^{j}\right) \notin\left(\underline{r}^{-}, \underline{r}^{+}\right)$.

By [RS2, Proposition 7.4 and Lemma 3.9], $\underline{r}^{-}$and $\underline{r}^{+}$are the characteristic addresses of some hyperbolic component $W^{\prime}$ with $W \subset \mathcal{W}\left(W^{\prime}\right)$. By (b) and the fact that the vertical order of parameter rays coincides with the lexicographic order on external addresses,

$$
\mathcal{W}\left(W^{\prime}\right) \cap\left(\bigcup_{k \geq 0, j \in\{1,2\}} \mathcal{G}_{\sigma^{k}\left(\underline{s}^{j}\right)}\right)=\emptyset .
$$

By Theorem 3, for each $j \in\{1,2\}$ and every $\kappa \in \mathcal{W}(W) \subset \mathcal{W}\left(W^{\prime}\right)$, the ray $g_{\underline{s}^{j}}^{\kappa}$ lands at a periodic point $z^{j}(\kappa)$, which depends holomorphically on $\kappa$. The maps $z^{1}$ and $z^{2}$ are both analytic extensions of the periodic point $z_{0}$, and must therefore be equal, as required. 


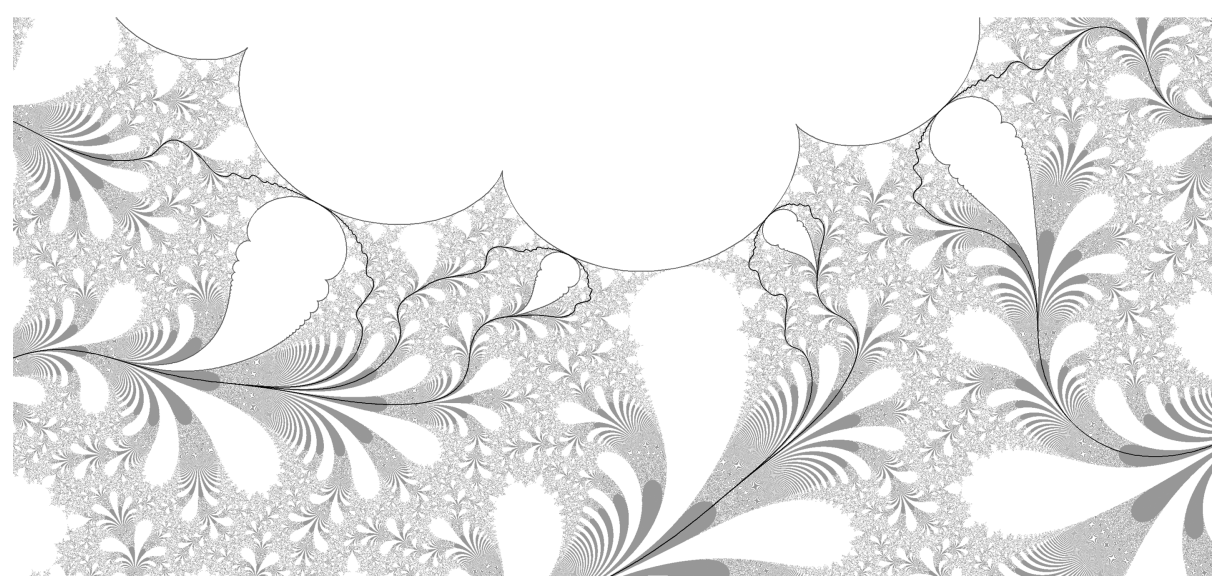

Figure 2. Some subwakes of the period 5 component from Figure 1

Suppose that $W$ is a hyperbolic component and $V$ is a hyperbolic component of higher period such that $\partial V \cap \partial W \neq \emptyset$. Then $V$ is called a child component of $W$, and $\mathcal{W}(V)$ is called a subwake of $W$ (compare Figure 2).

Proposition 6. Let $W$ be a hyperbolic component and let $V$ be a child component of $W$. Then $\partial W \cap \partial V$ consists only of the root $\kappa_{1}$ of $V$.

Furthermore, let $\kappa_{0} \in W$, let $z_{0}$ be a point on the attracting orbit of $E_{\kappa_{0}}$ and let $U$ be an open neighborhood of $W \cup\left\{\kappa_{1}\right\}$ such that $U \cap \mathcal{W}(V)$ is connected and $z_{0}$ has an analytic extension $z$ to $U$. Then $z$ extends analytically to $U \cup \mathcal{W}(V)$ and $z(\kappa)$ is the landing point of at least two periodic dynamic rays for every $\kappa \in \mathcal{W}(V)$.

If $W^{\prime} \neq W$ is a hyperbolic component with $W^{\prime} \subset \mathcal{W}(W)$, then $W^{\prime}$ is contained in some subwake of $W$.

Proof. By [RS1, Proposition 8.1], $\partial W \cap \partial V$ contains only parabolic parameters. By [RS2, Proposition 5.1 and Theorem 5.3], no parabolic parameter except $\kappa_{1}$ can belong to this intersection, and there are at least two periodic rays landing at each point of the parabolic orbit of $E_{\kappa_{1}}$. The point $z(\kappa)$ belongs to this parabolic orbit when $\kappa=\kappa_{1}$, and becomes repelling when $\kappa$ is perturbed into $V$.

By [RS2, Proposition 5.2], the point $z(\kappa)$ is still the landing point of at least two periodic rays after such a perturbation, and the second statement now follows from Proposition 5, The final claim is [RS2, Corollary 6.9].

Finally, we shall require the following bound for parameters on parameter rays with large combinatorics [R2, Corollary 4.8]. (Also compare [RS1, Lemma 5.10].)

Proposition 7. Suppose that $\underline{s}$ is an external address of period $n$, and suppose that $M:=\max \left|s_{k}\right|>F^{n}(6)$. Then

$$
G_{\underline{s}} \subset\left\{\kappa:|\kappa|>\frac{1}{5} F^{-(n-1)}(2 \pi M)\right\} .
$$




\section{Proof of Theorem 2}

Theorem 4. Let $E_{\kappa_{0}}$ be an exponential map for which the singular value does not escape. Suppose that $E_{\kappa_{0}}$ has a periodic point $z_{0}$ of period $n$ which is not the landing point of a periodic dynamic ray. Then there exists a hyperbolic component $W$ of period $n$ such that $\kappa$ lies in $\mathcal{W}(W)$, but not in any subwake of $W$.

Furthermore, all period $n$ points of $E_{\kappa_{0}}$ which do not lie on the orbit of $z_{0}$ are landing points of periodic dynamic rays.

Proof. By Proposition 2 we can suppose that $\kappa_{0}$ is not a parabolic parameter. Consider the set $A$ consisting of all parameter rays of period dividing $n$ together with the landing points of these rays; by Proposition 3 , the latter points are exactly the parabolic parameters whose ray period divides $n$. We claim that $A$ is closed. Indeed, for every $M \in \mathbb{N}$ the finite union

$$
A_{M}:=\bigcup\left\{\overline{G_{\underline{s}}}: \underline{s} \text { is periodic of period dividing } n \text { and }\left|s_{j}\right| \leq M \text { for all } j\right\}
$$

is closed. By Proposition 7, the sequence of sets $\left(A_{M} \backslash A_{M-1}\right)$ has no finite accumulation point as $M \rightarrow \infty$. Thus $A=\bigcup A_{M}$ is closed as well.

Now let $U$ be the component of $\mathbb{C} \backslash A$ which contains $\kappa_{0}$. By Proposition 4 , $U$ contains a unique hyperbolic component $W$ of period $k \mid n$, and this component satisfies $U \subset \mathcal{W}(W)$. Since $A \cup\{\infty\}$ is a connected subset of the Riemann sphere, $U$ is simply connected. Thus any period $n$ point $z$ of $E_{\kappa_{0}}$ has an analytic extension $z: U \rightarrow \mathbb{C}$. Let $\kappa \in W$. We claim that if $z$ is not the landing point of a periodic dynamic ray of $E_{\kappa_{0}}$, then $z(\kappa)$ is on the attracting orbit of $E_{\kappa}$ (and in particular, $k=n)$. Indeed, suppose that $z(\kappa)$ is not on the attracting orbit of $E_{\kappa}$; then it is a repelling periodic point of $E_{\kappa}$. By Proposition 2, there is a periodic dynamic ray $g_{\underline{s}}^{\kappa}$ landing at $z(\kappa)$. If more than one periodic ray lands at $z(\kappa)$, then by Proposition 5. $g_{\underline{s}}^{\kappa_{0}}$ lands at $z$. Otherwise, $\underline{s}$ has period $n$. Because $U$ does not intersect any parameter rays of period $n$, Theorem 3 implies that, again, $g_{\underline{s}}^{\kappa_{0}}$ lands at $z$.

So the analytic extension of $z_{0}$ throughout $U$ becomes attracting in $W$, and every period $n$ point which is not on the orbit of $z_{0}$ is the landing point of a periodic dynamic ray of $E_{\kappa_{0}}$. If $V$ is a child component of $W$, then $\mathcal{W}(V) \cap U$ is clearly connected. Thus $\kappa \notin \mathcal{W}(V)$ by Proposition 6 .

Proof of Theorem 2, Let $z$ and $w$ be periodic points of $E_{\kappa}$ which lie on two different periodic orbits and let $n$ and $m$ be their respective periods. Suppose by contradiction that neither point is the landing point of a periodic dynamic ray of $E_{\kappa}$. By the previous theorem, $n \neq m$ and there exist period $n$ and $m$ hyperbolic components $W$ and $V$ with $\kappa \in \mathcal{W}(W) \cap \mathcal{W}(V)$ so that $\kappa$ is not contained in any subwake of $W$ or $V$. Because the two wakes are not disjoint, one is contained in the other; suppose without loss of generality that $\mathcal{W}(W) \subset \mathcal{W}(V)$. By Proposition 6. $\mathcal{W}(W)$ lies in a subwake of $V$, which is a contradiction.

By Theorem 4 , the conjecture that every repelling periodic point of an exponential map with nonescaping singular value is the landing point of a periodic dynamic ray is equivalent to the following conjecture about parameter space. (For a discussion, see [R1, Section 7.2].)

Conjecture. Let $W$ be a hyperbolic component. Then $\mathcal{W}(W)$ is the union of $\bar{W}$, the subwakes of $W$, and certain parameter rays. 


\section{ACKNOWLEDGMENTS}

I thank Walter Bergweiler, Markus Förster and Dierk Schleicher, as well as the audiences of the seminars at Kiel, Nottingham, Open University, Warwick and the MPI Bonn for interesting discussions on this work. Much appreciation is owed to Misha Lyubich, John Milnor and the Institute of Mathematical Sciences at Stony Brook for continued support and encouragement. I also thank the University of Warwick, where part of this work was conducted while holding a Marie Curie Studentship, for its hospitality.

\section{REFERENCES}

[BR] I. Noël Baker and Philip J. Rippon, Iteration of exponential functions, Ann. Acad. Sci. Fenn. Ser. A I Math. 9 (1984), 49-77. MR0752391 (86d:58065)

[BDD] Ranjit Bhattacharjee, Robert L. Devaney, R. E. Lee Deville, Krešimir Josić, and Monica Moreno-Rocha, Accessible points in the Julia sets of stable exponentials, Discrete Contin. Dyn. Syst. Ser. B 1 (2001), no. 3, 299-318. MR.1849820 (2002m:37058)

[BDG] Clara Bodelón, Robert L. Devaney, Michael Hayes, Gareth Roberts, Lisa R. Goldberg, and John H. Hubbard, Hairs for the complex exponential family, Internat. J. Bifur. Chaos Appl. Sci. Engrg. 9 (1999), no. 8, 1517-1534. MR1721835(2001a:37055)

[DGH] Robert L. Devaney, Lisa R. Goldberg, and John H. Hubbard, A dynamical approximation to the exponential map by polynomials, Preprint, MSRI Berkeley, 1986; compare [BDG].

[DH] Adrien Douady and John Hubbard, Etude dynamique des polynômes complexes, Prépublications mathémathiques d'Orsay (1984 / 1985), no. 2/4.

[EL] Alexandre È. Erëmenko and Mikhail Yu. Lyubich, Dynamical properties of some classes of entire functions, Ann. Inst. Fourier (Grenoble) 42 (1992), no. 4, 989-1020. MR 1196102 (93k:30034)

[F] Markus Förster, Parameter rays for the exponential family, Diplomarbeit, Techn. Univ. München, 2003, Available as Thesis 2003-03 on the Stony Brook Thesis Server.

[FRS] Markus Förster, Lasse Rempe, and Dierk Schleicher, Classification of escaping exponential maps, Preprint, 2004, arXiv:math.DS/0311427, submitted for publication.

[FS] Markus Förster and Dierk Schleicher, Parameter rays for the exponential family, Preprint, 2005, arXiv:math.DS/0505097.

[MSS] Ricardo Mañé, Paulo Sad, and Dennis Sullivan, On the dynamics of rational maps, Ann. Sci. École Norm. Sup. (4) 16 (1983), no. 2, 193-217. MR0732343 (85j:58089)

[M1] John Milnor, Dynamics in one complex variable, Friedr. Vieweg \& Sohn, Braunschweig, 1999, arXiv:math.DS/9201272. MR.1721240 (2002i:37057)

[M2] - Periodic orbits, externals rays and the Mandelbrot set: an expository account, Astérisque (2000), no. 261, xiii, 277-333, Géométrie complexe et systèmes dynamiques (Orsay, 1995), arXiv:math.DS/9905169. MR1755445(2002e:37067)

[R1] Lasse Rempe, Dynamics of exponential maps, doctoral thesis, Christian-AlbrechtsUniversität Kiel, 2003, http://e-diss.uni-kiel.de/diss_781/.

[R2] - Topological dynamics of exponential maps on their escaping sets, Preprint, 2003, arXiv:math.DS/0309107, conditionally accepted for publication in Ergodic Theory Dynam. Systems.

[R3] , Siegel disks and periodic rays of entire functions, Preprint, 2004, arXiv:math.DS/0408041, submitted for publication.

[R4] - Nonlanding dynamic rays of exponential maps, Preprint, 2005, arXiv: math.DS/0511588, submitted for publication.

[R5] - On entire functions with accessible singular value, in preparation.

[RS1] Lasse Rempe and Dierk Schleicher, Bifurcations in the space of exponential maps, Preprint \#2004/03, Institute for Mathematical Sciences, SUNY Stony Brook, 2004, arXiv:math.DS/0311480, submitted for publication.

[RS2] - Combinatorics of bifurcations in exponential parameter space, Preprint, 2004, arXiv:math.DS/0408011, submitted for publication.

[S1] Dierk Schleicher, On the dynamics of iterated exponential maps, Habilitation thesis, TU München, 1999. 
[S2] Attracting dynamics of exponential maps, Ann. Acad. Sci. Fenn. Math. 28 (2003), 3-34. MR1976827 (2004k:37091)

[SZ1] Dierk Schleicher and Johannes Zimmer, Escaping points of exponential maps, J. London Math. Soc. (2) 67 (2003), no. 2, 380-400. MR1956142 (2003k:37067)

[SZ2] Periodic points and dynamic rays of exponential maps, Ann. Acad. Sci. Fenn. Math. 28 (2003), 327-354. MR.1996442 (2004e:37068)

Mathematics Institute, University of Warwick, Coventry CV4 7AL, United Kingdom

E-mail address: lasse@maths.warwick.ac.uk

$U R L:$ http://www . maths . warwick.ac.uk/ lasse

Current address: Department of Mathematical Sciences, The University of Liverpool, Liverpool L69 7ZL, United Kingdom 